\title{
Magnetic Inflation of Magnetic Plasma Sail by One Component Plasma Simulation
}

\author{
By Daisuke AKITA ${ }^{1,4)}$, Hiroko UEDA ${ }^{2,4)}$, Iku SHINOHARA ${ }^{2,4)}$, Ikkoh FunAKI ${ }^{2,4)}$ and Hideyuki UsuI ${ }^{3,4)}$ \\ ${ }^{1)}$ Department of International Development Engineering, Tokyo Institute of Technology, Tokyo, Japan \\ ${ }^{2}$ Institute of Space and Astronautical Science, Japan Aerospace Exploration Agency, Sagamihara, Japan \\ ${ }^{3)}$ Department of Computer Science and Systems Engineering, Kobe University, Kobe, Japan \\ 4) Japan Science and Technology Agency (JST), CREST, Kawaguchi, Saitama, Japan
}

(Received July 17th, 2009)

\begin{abstract}
Magnetic plasma sails have possibilities to reduce drastically mission durations of deep space explorations by using the momentum of the solar wind to produce its thrust. In order to produce a practical magnitude of the thrust, the magnetic plasma sails are required to inflate applied magnetic fields by injecting plasma from the spacecrafts. In this study, the magnetic field inflation by the plasma injection is numerically simulated by one component plasma model, which can be performed more robustly than those by hybrid simulations. The fundamental features of the injected plasma flow field and the electromagnetic field around the magnetic plasma sail are investigated, especially on the effects of the direction and the density of the plasma injection. The magnetic field can be inflated by the plasma injection in the one component plasma model. The direction and density of the plasma injection have strong impacts on the flow field and magnetic field inflation.
\end{abstract}

Key Words: Magnetic Plasma Sail, Magnetic Inflation, Plasma Simulation

\section{Nomenclature}

$\begin{array}{cll}\boldsymbol{V} & : \text { mean velocity, } \mathrm{m} / \mathrm{s} \\ \boldsymbol{v} & : & \text { thermal velocity, } \mathrm{m} / \mathrm{s} \\ n & : & \text { number density, } / \mathrm{m}^{3} \\ m & : & \text { ion mass, } \mathrm{kg} \\ q & : & \text { ion charge, } \mathrm{c} \\ r & : & \text { radius, } \mathrm{m} \\ \boldsymbol{J} & : & \text { current density, } \mathrm{A} / \mathrm{m}^{2} \\ \boldsymbol{B} & : & \text { magnetic flux density, } \mathrm{T} \\ \boldsymbol{E} & : & \text { electric field, } \mathrm{V} / \mathrm{m} \\ \boldsymbol{A} & : & \text { vector potential, Tm } \\ \mu_{0} & : \text { magnetic permeability, } \mathrm{N} / \mathrm{A}^{2} \\ \beta & : & \text { ratio of dynamic and magnetic pressure } \\ \text { Subscripts } & & \\ 0 & : & \text { coil } \\ L & : & \text { Larmor motion } \\ T & : & \text { transverse (divergence-free) component }\end{array}$

\section{Introduction}

Advanced in-space propulsion systems, which can reduce drastically the mission durations and the operational costs, are necessary to increase opportunities of deep space explorations. In addition, conventional propulsion systems, which take a long duration to reach the deep space, may have difficulties to expand the exploration region, since the onboard instruments and batteries have a limited lifetime.

Magnetic sails, which are proposed by Zubrin ${ }^{1)}$, are attractive in-space propulsion concepts. The magnetic sail uses the momentum of the solar wind ${ }^{2)}$ to produce its thrust. Since the momentum flux originating from the sun is used to accelerate the spacecraft like a solar sail, the magnetic sail does not require onboard propellant. The solar wind is a high-speed rarefied plasma flow mainly composed of protons and electrons. The magnetic sail produced the thrust by the interaction of the ambient solar wind with the magnetic field artificially applied around the spacecraft. The artificial magnetic field is applied by a coil current, which is provided by the power system of the spacecraft. If the solar wind blows with some relative velocity to the spacecraft, the solar wind is deflected by the artificial magnetic field applied by the coil current. The momentum change of the solar wind produces the drag force on the magnetic sail in the direction to the incoming solar wind. The drag force continuously accelerates the spacecraft in the direction of the incoming solar wind relative to the spacecraft's motion. The maximum available speed of the magnetic sail would be that of the solar wind itself.

However, the magnetic sail, as well as a solar sail, requires a huge sail to produce a practical magnitude of the thrust for deep space explorations, since the density of the solar wind is very low. Figure 1 shows the comparison between the dynamic pressure of the solar wind and the solar radiation pressure, which drive the magnetic sail and the solar sail, respectively, as a function of the distance from the sun. Both the pressures decrease inversely proportional to the square of the distance from the sun. The dynamic pressure of the solar wind is much smaller than the radiation pressure by three orders of magnitude at the earth's orbit. Therefore, if the magnetic sail can generate the magnetic field whose diameter is dozens of times larger than that of the solar sail's foil, the magnetic sail would produce the same magnitude of the thrust as the solar sail. The magnetic sail uses the magnetic field for the sail, so that a huge coil current is not necessarily required 


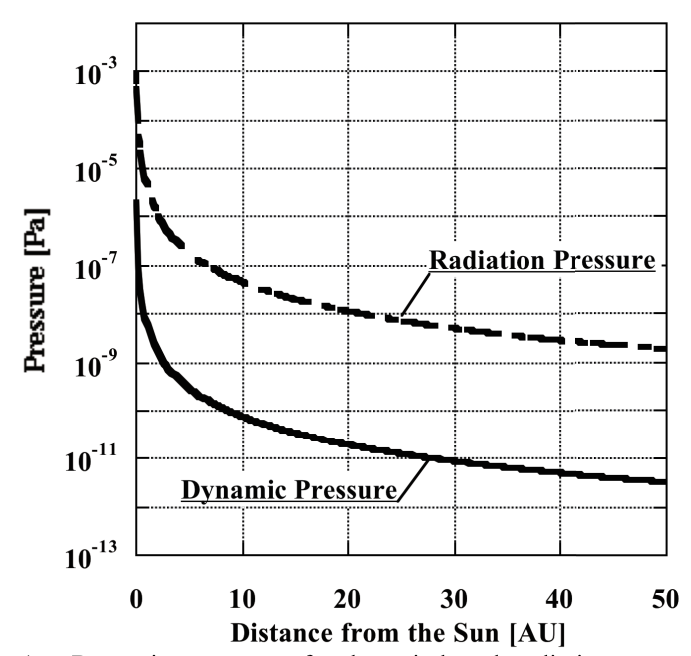

Fig. 1. Dynamic pressure of solar wind and radiation pressure for distance from the sun.

to generate the huge magnetic field. However, it is difficult to generate such a huge magnetic field only by the coil current under the present state of electromagnetic technologies.

Mini-Magnetospheric Plasma Propulsion (M2P2) by Winglee $^{3)}$, also called Magnetic Plasma Sail (MPS), has a possibility to generate the huge magnetic field. Winglee proposed to inject plasma from the spacecraft into the original applied magnetic field, so as to inflate the original magnetic field. If the original magnetic field can be inflated by the plasma injection, the magnetic plasma sail would extend the interaction region of the magnetic field with the solar wind. The extended interaction region by the magnetic plasma sail could produce a sufficient magnitude of the thrust for the deep space missions.

The feasibility of the magnetic field inflation by the plasma injection has been studied by MHD and hybrid simulations ${ }^{4)}$. The magnetic field inflation and the interaction with the solar wind at far field from the spacecraft, where the particulate properties of the plasma become significant, are important for the performance evaluation of the magnetic plasma sail. Nevertheless, MHD simulation cannot evaluate the particulate properties of the plasma. While the conventional hybrid simulation can evaluate the particulate properties of the plasma ion, it is difficult to simulate the significant gradient of the density between the injected dense plasma and the ambient rarefied plasma in terms of the simulation methodology. In addition, it is required that the dynamic pressure of the injected plasma is as low as possible for the efficient inflation of the magnetic field, because the injected plasma of the magnetic plasma sail is regarded as a propellant as in the case of electric propulsions. In the MHD or the hybrid simulation, however, it is also difficult to simulate the plasma injection whose density is sufficiently low in terms of computational costs.

In this study, the magnetic field inflation of the magnetic plasma sail is numerically simulated by one component plasma model ${ }^{5,6)}$, which can be relatively robustly performed under the above conditions including the particulate properties of the plasma ion. In the one component plasma model, only the plasma ions are regarded as particles under the quasi-neutral approximation. The simulation can be performed robustly with a computational cost comparable to the hybrid simulation, while only the cases that the relatively slow physical processes are significant are available.

The fundamental features of the injected plasma flow and the electromagnetic field around the magnetic plasma sail are investigated, especially on the effects of the direction and the density of the plasma injection.

\section{Numerical Simulation Method}

\subsection{One component plasma model ${ }^{5,6)}$}

The one component plasma model, which regards only the plasma ions as particles, neglects displacement currents and the longitudinal (rotation-free) component of the electric field under the quasi-neutral approximation. In this study, we are interested in the temporal change in the magnetic field, which depends only on the transverse (divergence-free) component of the ion current. Under the above assumption, therefore, it is assumed that the ion's motion makes the transverse component of the ion current and the longitudinal component of the ion current is implicitly compensated by the electron current.

Figure 2 shows the flowchart of the one component plasma simulation. The simulation consists mainly of the calculation of the sample particles' motion and that of the induced electromagnetic field. The initial particle velocity of the injected plasma is stochastically given by assuming the Maxwellian distribution at the mean velocity and the temperature. The charge and current densities at the respective computational nodes are calculated from the instantaneous position and velocity of the sample particles by the PIC weighting interpolation. The electromagnetic field is calculated from the above charge and current density by solving the following Poisson equations derived from Maxwell equations under the above assumption. The magnetic field is obtained from the vector potential, which is written by:

$$
\nabla^{2} \mathbf{A}_{\mathrm{T}}=-\mu_{0} \mathbf{J}_{\mathrm{T}} .
$$

Differentiating Eq. (1) with respect to time, the transverse component of the electric field is given by:

$$
\nabla^{2} \mathbf{E}_{\mathbf{T}}=\mu_{0} \mathbf{J}_{\mathrm{T}} .
$$

There are some techniques for obtaining $\dot{\mathbf{J}}_{\mathrm{T}}$ (see ref. 5, 6 for details). The Lorenz force at each particle's position is interpolated from the electromagnetic field obtained at the computational node. The equation of motion of each sample particle is numerically integrated and then the position and velocity are updated for all the sample particles. The updated particle's position and velocity are used to update the calculation of the induced electromagnetic field. In order to obtain the good convergence in the solution of the flow field and electromagnetic field, a sufficiently large number of time stepping are made before the time-averaged properties are calculated. The time-averaged properties are calculated for the sufficiently long time to average the fluctuations of the field properties due to the statistical oscillation. All the results of the field properties in the following sections are thus the time-averaged properties. 


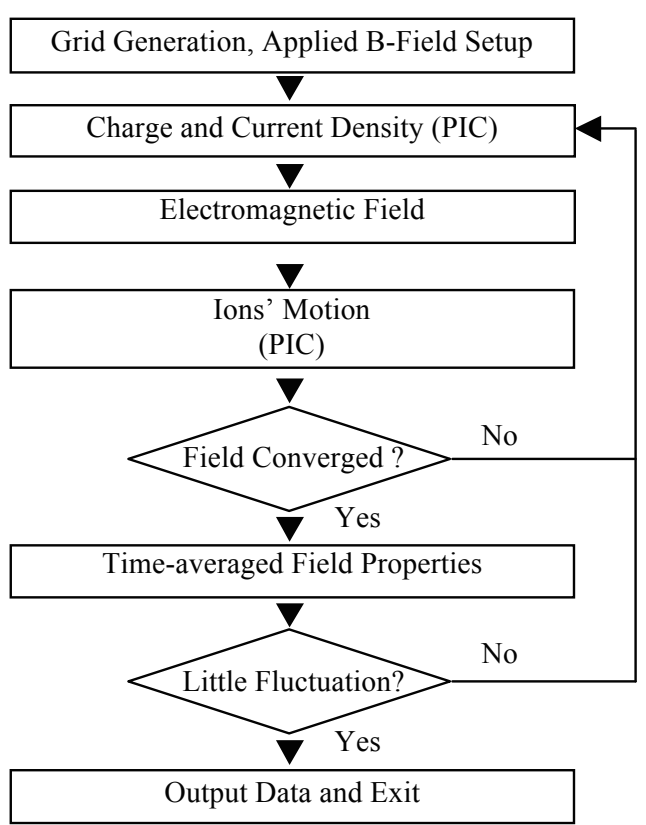

Fig. 2. Flowchart of one component plasma simulation.

The inter-particle collision is not considered, since the mean free path of the injected plasma ion is larger than the representative length supposed here. The time step size of the simulation is restricted by CFL condition for Alfven speed and by the Larmor frequency of the ions.

\subsection{Numerical conditions}

Numerical conditions are assumed by reference to the previous studies. The computational domain has an area of $160 \mathrm{~km} \times 160 \mathrm{~km} \times 160 \mathrm{~km}$ composed of equally-spaced $51 \times$ $51 \times 51$ nodes $(132,651$ in total) as shown in Fig. 3. The coil current of about $18.5 \mathrm{kAturn}$, which represents the magnetic plasma sail, is located at the center of the computational domain. The coil radius is $r_{0}=2 \mathrm{~km}$ and the magnetic flux density is $B_{0}=5.8 \times 10^{-6} \mathrm{~T}$ at the center of the coil. The injected plasma is assumed to be Ar whose mean velocity, the number density and the temperature are $V=40 \mathrm{~km} / \mathrm{s}, n=10^{8}-10^{10} / \mathrm{m}^{3}$ and $1.0 \mathrm{eV}$, respectively. The number density of the injected plasma is set to be relatively low in order to investigate the magnetic inflation that is difficult to be computed by hybrid simulations. The plasma is symmetrically injected into the vacuum from the position just outside the coil. The mean velocity of the injected plasma has only the one component, which is same to the direction of the plasma injection. The Larmor radius $r_{L}$ for the mean velocity of the injected plasma is comparable to the coil radius $r_{0}$ at the center of the coil. The ratio of the dynamic pressure of the injected plasma and the magnetic pressure of the coil at the center of the coil is $\beta=\left(m n V^{2} / 2\right) /\left(B_{0}^{2} / 2 \mu_{0}\right)=10^{-3}-10^{-5}$. In the present study, two cases regarding the direction of the plasma injection are performed: a) the direction of the plasma injection is parallel to the magnetic moment vector of the coil (i.e. injection from the polar), b) the direction of the plasma injection is

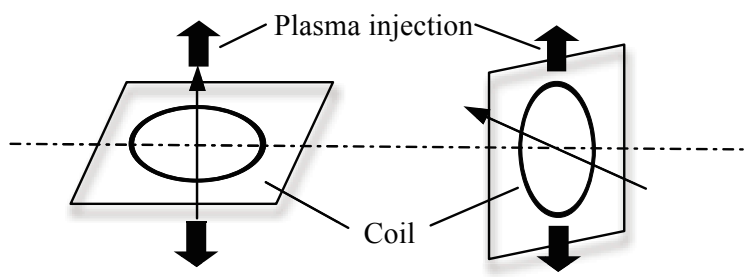

a) Injection from polar

b) Injection from equator

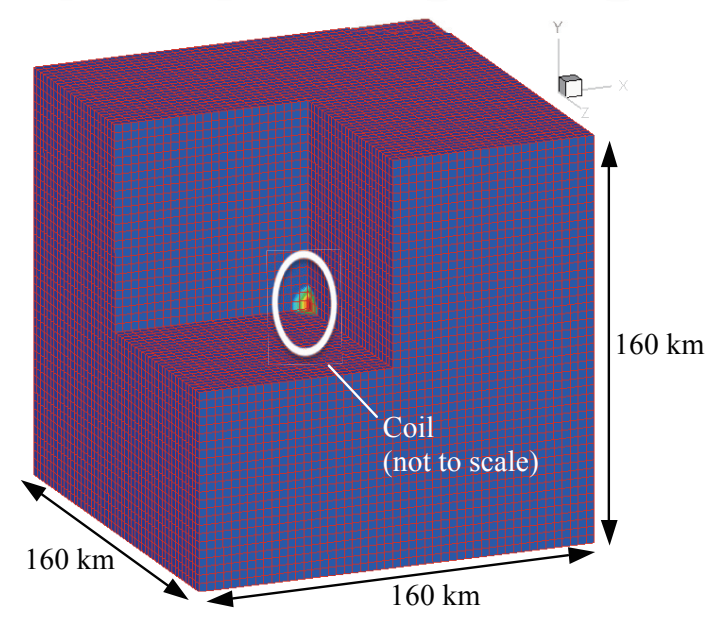

Fig. 3. Grid system and the coil current configuration.

perpendicular to the magnetic moment vector of the coil (i.e. injection from the equator), as illustrated in Fig. 3 a) and b), respectively.

\section{Results and Discussions}

\subsection{Injection from the polar}

Figure 4 shows the number density distribution of the injected plasma ion on the plane involving the magnetic moment vector of the coil. The contour in Fig. 4 is described in exponential scale. The number density of the injected plasma ion is $n=10^{10} / \mathrm{m}^{3}$. The number density distribution is almost axisymmetric around the magnetic moment axis of the coil. The upward streamlines of the ion, which is not indicated in Fig. 4, are curved by the applied magnetic field in the clockwise direction as viewed from the coil in the upper region of the coil. In the lower region of the coil, the downward streamlines are curved in the counterclockwise direction as viewed from the coil. This means that the streamlines of the injected plasma ion are curved in the same direction around the magnetic moment axis of the coil in both the upper and lower regions of the coil, which is the same direction of the coil current.

In this case, the interaction strength between the injected plasma ion and the magnetic field could be evaluated by the ratio of the ion travel distance in the direction parallel to the magnetic field during the Larmor period $D=2 \pi m V / q B$ and the Larmor radius $r_{L}=m v / q B$. The Larmor radius is calculated by using the thermal velocity $v$, which is perpendicular to the magnetic field. The ratio $D / r_{L}$ is proportional only to the ratio of the mean velocity and the thermal velocity. In this case, the ratio $D / r_{L}$ is relatively large, so that the interaction strength 


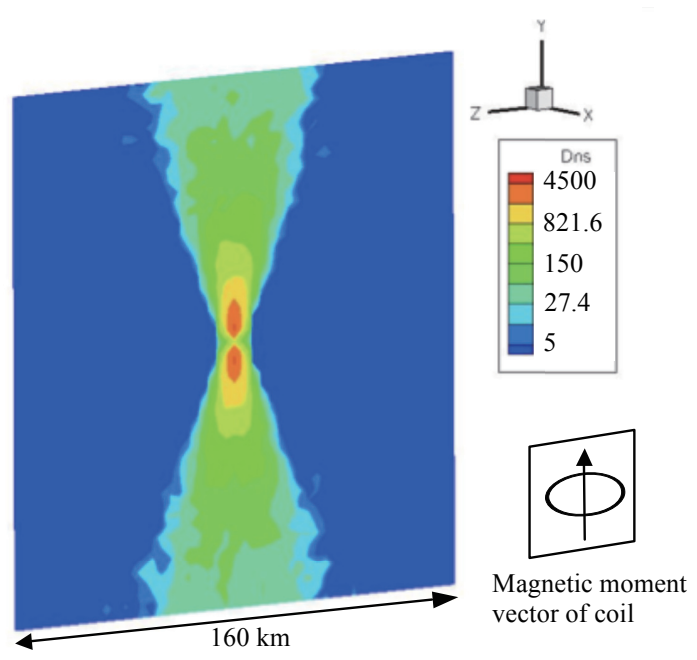

Fig. 4. Number density distribution of injected plasma ion (injection from the polar, $n=10^{10} / \mathrm{m}^{3}$ ).

between the injected plasma ion and the magnetic field is weak. In contrast, when the ratio $D / r_{L}$ is relatively small, the interaction would be similar to the behavior in a case of MHD.

Figure 5 shows the normalized magnetic flux density distribution in the same case of Fig. 4. Figure 6 shows the initial magnetic flux density distribution before the plasma injection. The contour in Fig. 5 and Fig. 6 are also described in exponential scale. By comparing Fig. 5 and Fig. 6, the magnetic flux density is increased in both the upper and lower regions of the coil, since the streamlines of the injected ion, which makes the induced current, is curved in the same direction of the coil current as mentioned above. However, the induced current does not increase the magnetic flux density microscopically everywhere in the computational domain.

In Fig. 5, the change in the magnetic flux density is relatively small in the region along the magnetic moment axis of the coil. That would be caused by the small angle between

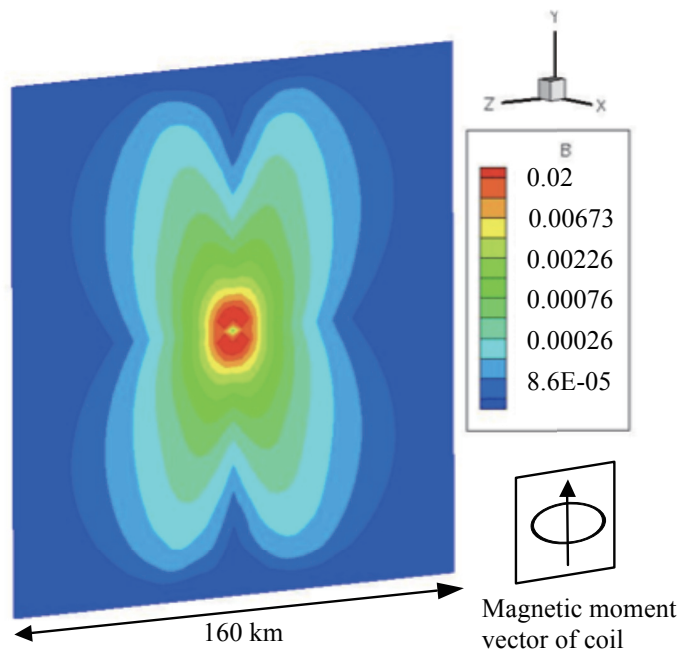

Fig. 5. Magnetic flux density distribution (injection from the polar, $\left.n=10^{10} / \mathrm{m}^{3}\right)$.

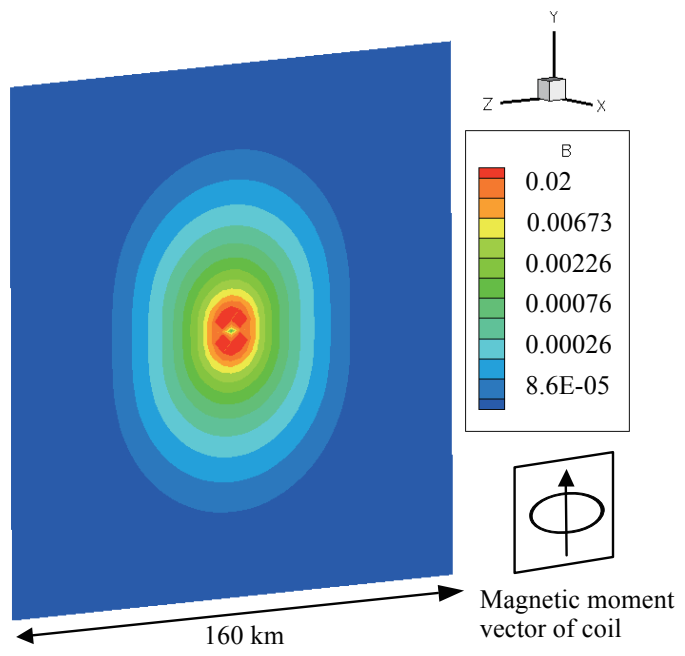

Fig. 6. Initial magnetic flux density distribution before the plasma injection (injection from the polar).

the injected ion velocity vector $\boldsymbol{V}$ and the magnetic flux density vector $\boldsymbol{B}$, since the $\boldsymbol{V} \times \boldsymbol{B}$ makes the spiral streamlines and then the induced current around the magnetic moment axis of the coil.

Figure 7 shows the normalized magnetic flux density distribution on the magnetic moment axis of Fig. 5 for the distance from the coil center, compared with the distribution before the plasma injection. The distance from the coil center is normalized by the length on a side of the computational domain. The rate of decrease of the inflated magnetic field is approximately $B \propto r^{-2.6}$.

Figure 8 shows the magnetic flux density distribution on the centerline perpendicular to the magnetic moment axis of the coil in the case of Fig. 5. There is little difference by the plasma injection, since the number density of the injected plasma is very low in that region as seen in Fig. 4.

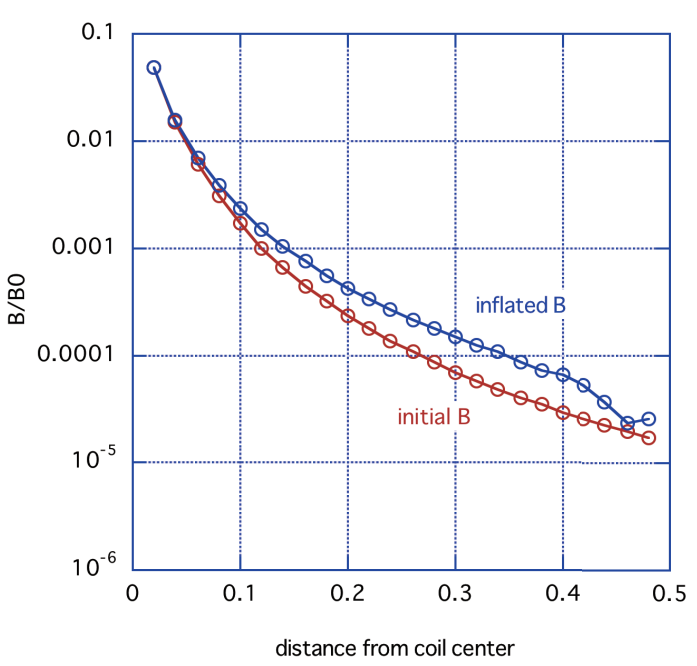

Fig. 7. Magnetic flux density distribution on the magnetic moment axis (injection from the polar, $n=10^{10} / \mathrm{m}^{3}$ ). 


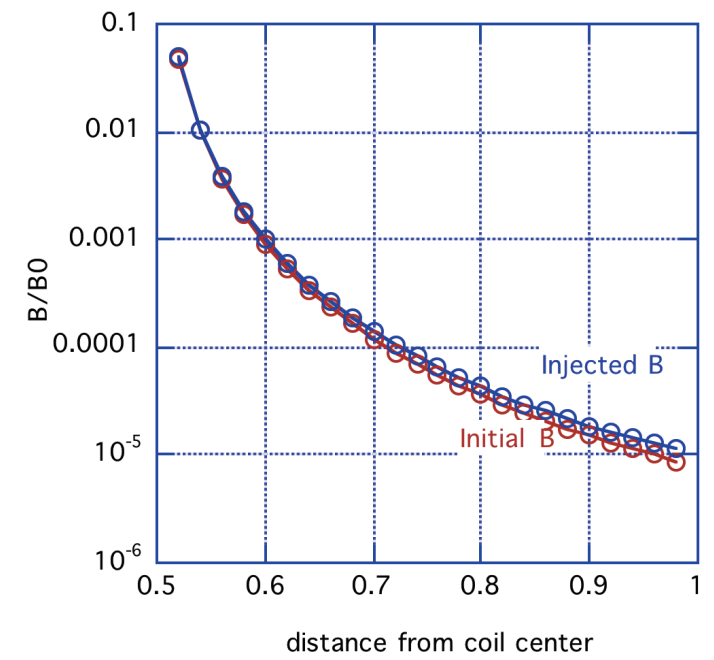

Fig. 8. Magnetic flux density distribution on the centerline perpendicular to the magnetic moment axis (injection from the polar, $\left.n=10^{10} / \mathrm{m}^{3}\right)$.

\subsection{Injection from the equator}

Figure 9 shows the number density distribution of the injected plasma ion on the plane of the coil. The number density of the injected plasma ion is $n=10^{10} / \mathrm{m}^{3}$. Although the flow field is more complicated than that in the case of the injection from the polar, the number density distribution is almost symmetric around the magnetic moment axis of the coil. The streamlines of the ion are curved by the applied magnetic field in the same direction of the coil current, which is the same behavior as the case of the injection from the polar. However, the representative Larmor radius is different from that in the case of the injection from the polar. In this case, the Larmor radius is evaluated by the mean velocity $V$ instead of the thermal velocity $v$, owing to the direction with respect to the magnetic field.

Figure 10 shows the normalized magnetic flux density distribution in the same case of Fig. 9. Figure 11 shows the

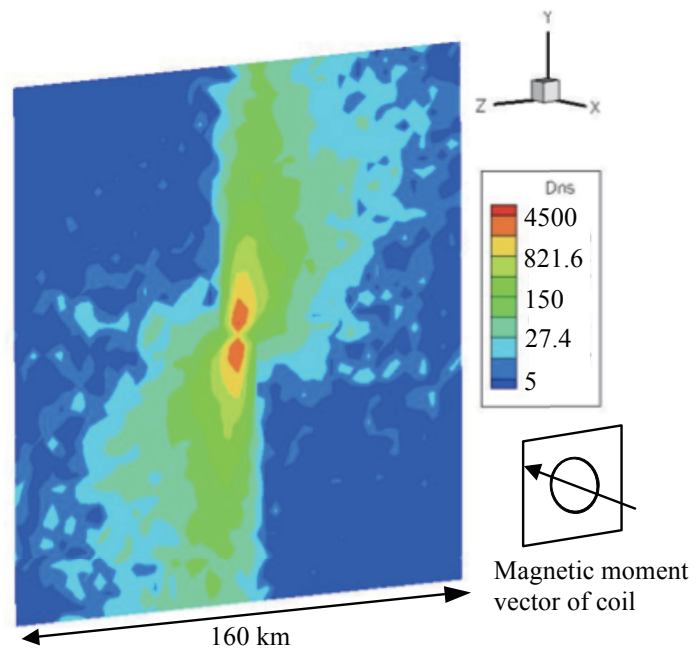

Fig. 9. Number density distribution of injected plasma ion (injection from the equator, $n=10^{10} / \mathrm{m}^{3}$ ).
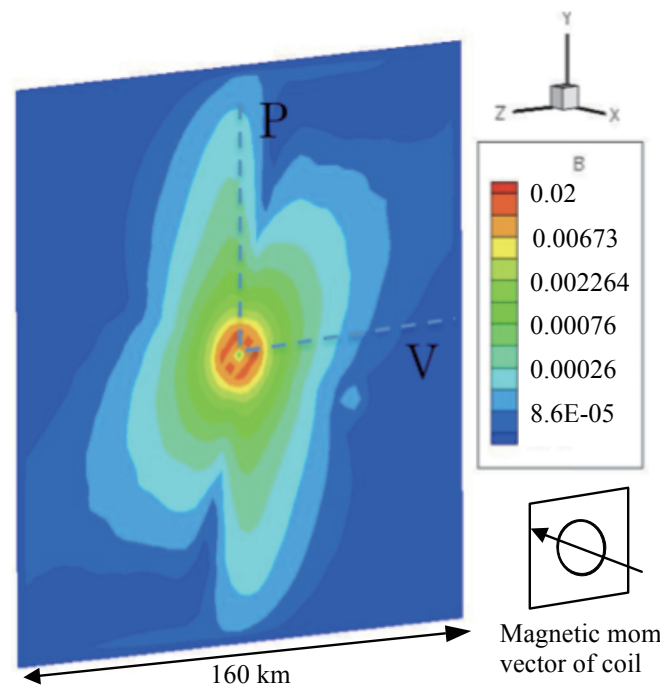

Magnetic moment vector of coil

Fig. 10. Magnetic flux density distribution (injection from the equator, $\left.n=10^{10} / \mathrm{m}^{3}\right)$.

normalized magnetic flux density distribution for the normalized distance from the coil center in the direction of the plasma injection, and in the direction perpendicular to the plasma injection and to the magnetic moment axis of the coil. $B_{P}$ and $B_{V}$ in the Fig. 11 are magnetic flux density on the line $\mathrm{P}$ and line $\mathrm{V}$ in Fig. 10. The magnetic flux density in the direction of the plasma injection is more increased than that in the perpendicular direction. It would be caused by the number density distribution shown in Fig. 9. In this case, the angle between the injected ion velocity vector $\boldsymbol{V}$ and the magnetic flux density vector $\boldsymbol{B}$ is almost perpendicular, so that the interaction of those would be more effective compared with that in the case of the injection from the polar.

Figure 12 shows the magnetic flux density distribution in the direction of the plasma injection whose number density is $10^{8} / \mathrm{m}^{3}$. The magnetic field inflation is vanishingly small, compared with the $B_{P}$ in Fig. 11. The flow field of the injected plasma ion is qualitatively similar to Fig. 9, although it is not

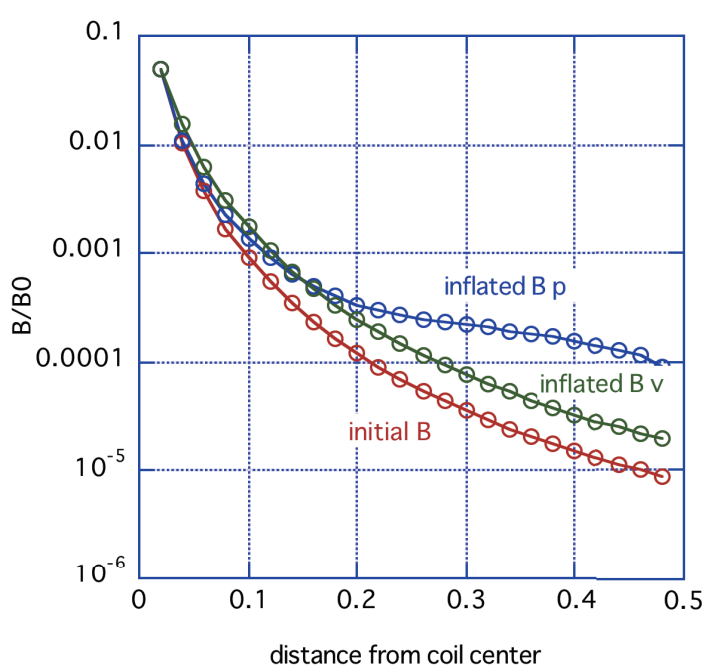

Fig. 11. Magnetic flux density distribution (injection from the equator, $\left.n=10^{10} / \mathrm{m}^{3}\right)$. 


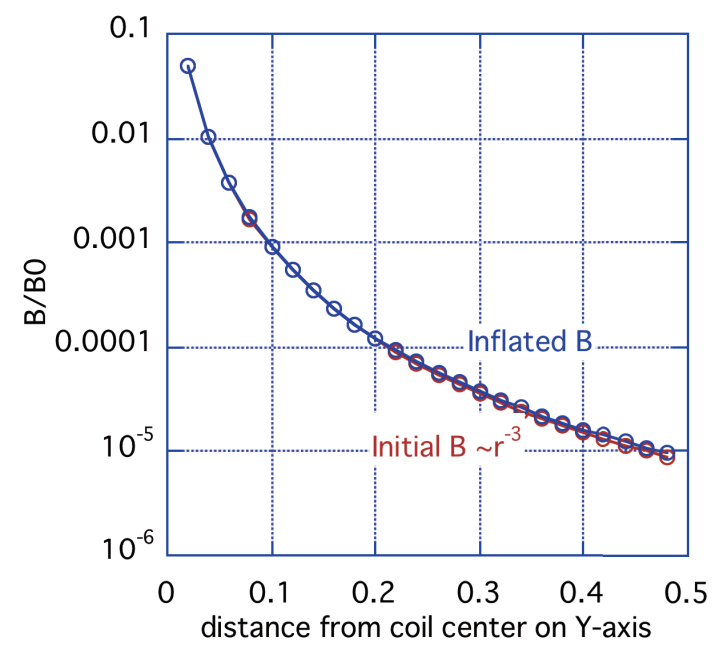

Fig. 12. Magnetic flux density distribution in the direction of the plasma injection (injection from the equator, $n=10^{8} / \mathrm{m}^{3}$ ).

shown. In the case of the number density of $10^{8} / \mathrm{m}^{3}$ and the injection from the polar, the effects of the plasma injection would be smaller than that of Fig. 12, comparing Fig. 7 with Fig.11.

\section{Summary}

The magnetic inflation of the magnetic plasma sail is numerically simulated by one component plasma model. The magnetic field can be inflated by the plasma injection in one component plasma model. Two cases with respect to the direction of the plasma injection are performed. The direction and density of the plasma injection have strong impacts on the flow field and magnetic field inflation. The streamlines of the injected plasma ion and thus the induced current, which is significantly affected by the conditions, would be important to understand the behavior of the magnetic field inflation. It is also confirmed that the plasma injection whose density is relatively low reduces the magnetic inflation. The behavior of the magnetic inflation would be significantly changed by various parameters other than investigated in this study, so that a wide range parameter survey will be necessary.

\section{Acknowledgments}

This research was supported by Core Research for Evolutional Science and Technology (CREST) program of Japan Science and Technology Agency (JST).

\section{References}

1) Zubrin, R. and Andrews, D.: Magnetic Sail and Interplanetary Travel, Journal of Spacecraft and Rocket, 282 (1991), pp.197-203.

2) Obayashi, T.: Space Science -Solar Terrestrial Physics-, Shokabo Publishing, 1970 (Japanese)

3) Winglee, R., et al.: Mini-Magnetospheric Plasma Propulsion Tapping the energy of the solar wind for spacecraft propulsion, Journal of Geophysical Research, 105 A9 (2000), pp.21067-21077.

4) Kajimura, Y., et al.: Quantitative Evaluation of Ion Kinetic Effect in Magnetic Field Inflation by Injection of Plasma Jet, J. Jpn. Soc. Aeronaut. Space Sci.(Japanese) (in press).

5) Nielson, C. and Lewis, H.: Particle-code models in the nonradiative limit, Methods Computational Phys, 16 (1976), pp. 367-388.

6) Busnardo-Neto, J., et al.: A Self-Consistent Magnetostatic Particle Code for Numerical Simulation of Plasma, J. Comp. Phys, 23 (1977), pp. 300-312. 